Polymer Journal, Vol. 39, No. 3, pp. 259-266 (2007)

(C) 2007 The Society of Polymer Science, Japan

\title{
Monte Carlo Simulation of a Model System for Ferroelectric Phase Transition in Polymers
}

\author{
Daisuke FuKUZAwA, Akihiro NishiokA, Tomonori KoDA, and Susumu IKEDA ${ }^{\dagger}$ \\ Faculty of Engineering, Yamagata University, 4-3-16 Jonan, Yonezawa 992-8510, Japan
}

(Received December 1, 2006; Accepted December 27, 2006; Published February 9, 2007)

\begin{abstract}
A model for ferroelectric phase transitions in copolymers of vinylidene fluoride (VDF) and trifluoroethylene (TrFE) has been examined using Monte Carlo (MC) simulation. The model assumes simple three-states which reflect structural variation of polymers in addition to the directional degrees of freedom of dipoles attached to molecular segments. Results of the simulation qualitatively supported theoretical interpretations obtained by the Bethe approximation of the model. Furthermore, microscopic details of the phase transition were revealed. Temperature dependences of spontaneous polarization and specific heat exhibit clearly a change in a thermodynamic order of the phase transition. The phase transition is classified by a ratio $n$ of intramolecular interaction to intermolecular interaction. The specific heat of the system becomes the maximum at the ratio $n$ where the temperature hysteresis of the phase transition vanishes. It shows that the phase transition is changed from the first order transition to the diffuse one through the critical second order phase transition. In addition, the transformation mechanisms are predicted. The system exhibiting the first order phase transition is transformed due to nucleation and growth. The system doing the diffuse transition is transformed due to spinodal decomposition. [doi:10.1295/polymj.PJ2006179]

KEY WORDS VDF-TrFE Copolymer / Monte Carlo Simulation / Ferroelectric Phase Transition / First Order Phase Transition / Diffuse Transition / Nucleation and Growth / Spinodal Decomposition /
\end{abstract}

Recent developments of organic functional materials let researchers recognize importance of the nonvolatile memory property of VDF-TrFE copolymers because thin films of the VDF-TrFE copolymers are easily produced by a solvent removal process and exhibit the excellent character of a ferroelectric memory device. ${ }^{1-3}$

The VDF-TrFE copolymers show the ferroelectricparaelectric phase transition at transition temperatures. ${ }^{4}$ Transition temperature and temperature hysteresis characterizing the phase transition depend on VDF ratio in the copolymer. Various experiments have concluded that the thermodynamic character of the phase transition is changed from the first order transition to the diffuse transition by a decrease in the VDF ratio. ${ }^{5}$ According to Takahashi et al., the copolymers including less than 47 mole \% of VDF exhibits antiferroelectricity in place of the ferroelectricity. However, in this case, the phase transition to paraelectricity also shows diffuseness although the ordered phase is antiferroelectric., ${ }^{6,7}$

In the ferroelectric phase, all-trans chains are packed regularly with electric dipoles along the direction perpendicular to the chain axis. The polarization made of oriented permanent dipoles is cooperatively reversed by an external electric field. In the paraelectric phase, a chain packed in the crystal system consists of random arrangement of $\mathrm{TG}^{+}, \mathrm{TG}^{-}, \mathrm{T}_{3} \mathrm{G}^{+}$ and $\mathrm{T}_{3} \mathrm{G}^{-}$isomers taking a random helix conforma- tion, where $\mathrm{T}$ and $\mathrm{G}$ stand for trans and gauche conformations, respectively, $\mathrm{T}_{3}$ expresses sequence of three T's and $\mathrm{G}^{+}$and $\mathrm{G}^{-}$are mirror images of each other. On the phase transition from ferroelectric to paraelectric, gauche conformations are created at random in chains of a trans sequence in addition to making a random packing of trans segments with dipoles of opposite directions.

Several trials of theoretical explanation have been given in order to understand this transition on the basis of the standard phase transition mechanisms. ${ }^{8,9} \mathrm{On}$ the other hand, Abe and Tashiro performed computer simulations of ferroelectric-paraelectric transformations using molecular dynamics focusing on the conformational change in polymer chains. Their simulations reproduced successfully relations between various interactions and material properties. ${ }^{10-12}$

Our previous reports explained the phase transition of the polymer in terms of the Bethe approximation of a simple three states model. ${ }^{13,14}$ The theory reproduced the ferroelectric phase transition. The model covered the transition phenomena changing from first order to diffuse transition. This paper is concerned with Monte Carlo (MC) simulation for this simple model. Since results obtained by approximation do not necessarily guarantee true solutions, the purpose of this simulation is to evaluate the approximation used in the previous report, specifically to confirm the first order phase transition. Furthermore, the rela-

${ }^{\dagger}$ To whom correspondence should be addressed (Tel/Fax: +81-238-26-3065, E-mail: ikeda@yz.yamagata-u.ac.jp). 
tion between the temperature hysteresis and the peak heights of specific heat is examined to elucidate the existence of the critical transition. Furthermore, transformation procedures during phase transition are investigated.

\section{MODEL AND MONTE CARLO METHOD}

Linear molecular segments in crystals of VDFTrFE copolymers take all-trans conformation or helix-like conformation. ${ }^{15}$ The all-trans conformation has permanent electric dipole moment that stems from the difference of electronic affinity between hydrogen and fluorine atoms. The helix-like segment consists of a mixture of trans and gauche conformations and does not have the net permanent dipole moment. The dipole moment of the all-trans conformation is perpendicular to the molecular chain axis. In the ferroelectric state of VDF-TrFE copolymers, dipoles of the planar zigzag chains align cooperatively to produce the spontaneous polarization of the material.

In order to represent such a situation, we adopt the three-states model which has been previously proposed to understand this phase transition. ${ }^{13,14}$ The model consists of two dimensional triangular lattice sites that have binomial states of the Ising model corresponding to all trans planar zigzag conformation and an added extra state corresponding to the helix-like conformation.

Interaction Hamiltonian of the system is written as

$$
H=-V \sum_{i>j}^{\prime} \sigma_{i} \sigma_{j}+W \sum_{i}\left(1-\sigma_{i}^{2}\right)
$$

where, $\sigma_{j}$ is the three-valued state variable of a segmental unit assigned to $j$-th lattice site. The $j$-th segmental unit takes the helix-like conformation when $\sigma_{j}=0 . \sigma_{j}=1$ and -1 correspond to planar zigzag conformations whose electric dipoles are opposite to each other. In eq 1 , the summation symbol with prime is for nearest neighbor sites, $V$ is the nearest neighbor intermolecular interaction, and $W$ is the intramolecular interaction discriminating different conformations of the segmental unit. Positive and negative values of $V$, respectively, favor parallel and anti-parallel configurations of neighboring dipoles attached to segments. We assume $V>0$, because we consider the ferroelectricity in low temperature region. Negative values of $V$ realize the antiferroelectricity of the ordered phase, although the thermodynamic character of the phase transition to the disordered phase is not changed as it is seen from eq 1 . Positive $W$ favors all-trans conformations $\sigma=1$ and $\sigma=-1$ and negative $W$ favors $\sigma=0$ for the helix-like conformation.

The Boltzmann factor of the present model system is described as

$$
\exp \left\{-\frac{V}{k T}\left[-\sum_{i>j}^{\prime} \sigma_{i} \sigma_{j}+n \sum_{i}\left(1-\sigma_{i}^{2}\right)\right]\right\}
$$

where $T$ is temperature, $k$ Boltzmann constant, and interaction ratio $n$ is

$$
n \equiv \frac{W}{V}
$$

Monte Carlo simulations are performed with the Metropolis algorithm ${ }^{16-18}$ for the model which takes degeneracy of the state $\sigma=0$ into consideration. The degeneracy comes from a variety of ways of including gauche conformations into trans chains. Denoting the number of the degeneracy of $\sigma=0$ by $Z$, we should choose, for one segment, one of $Z+2$ states that consist of two planar zigzag states of $\sigma=1$ and $\sigma=-1$ and $Z$ states of $\sigma=0$. In the following simulations, we assume $Z=10$ following the previous work. ${ }^{13}$

In the present study, number of segments, that is, number of lattice sites $N$ is set as $N=50 \times 50=$ 2500 in a usual periodic boundary condition. Unit of Monte Carlo Steps (MCS) consists of $N$ trials of changing $\sigma_{j}$ for randomly chosen $j$. After calculating $H_{\text {former }}$ the energy of the system in the present state, we try to change a chosen $\sigma_{j}$ to a candidate state that is randomly selected from $Z+1$ states that do not include the present $\sigma_{j}$. We calculate $H_{\text {candidate }}$ the energy of the system with the candidate $\sigma_{j}$. If $H_{\text {candidate }} \leqq$ $H_{\text {former }}$, the $\sigma_{j}$ is moved to the candidate state. If $H_{\text {candidate }}>H_{\text {former }}$, the $\sigma_{j}$ is moved to the candidate state in a probability $\exp \left[-\left(H_{\text {candidate }}-H_{\text {former }}\right) / k T\right]$. To do this, we use uniform random number $r$ of $0<r<1$. If $r<\exp \left[-\left(H_{\text {candidate }}-H_{\text {former }}\right) / k T\right]$, the $\sigma_{j}$ is changed to the candidate state, while on the other hand if $r>\exp \left[-\left(H_{\text {candidate }}-H_{\text {former }}\right) / k T\right]$, the candidate state is rejected and the former $\sigma_{j}$ is kept for the next state. We carry out $10^{7} \mathrm{MCS}$ for a temperature, and system properties at the temperature are averaged for not less than $5 \times 10^{6} \mathrm{MCS}$ after equilibration. For calculation the value of $V$ is taken as $1 \mathrm{kcal} / \mathrm{mole}$ although following results are not changed even if other values are taken.

\section{RESULTS AND DISCUSSION}

\section{Temperature Dependence of Polarization and Popula- tion Ratio of Each State}

The simulation is started at the lowest temperature with the initial state $\sigma_{j}=1$ for all $j$. In the heating (cooling) process, the initial state of the simulation at each temperature is an equilibrium state obtained in the preceding simulation at a lower (higher) temperature. We monitor polarization $P$ of the system: 


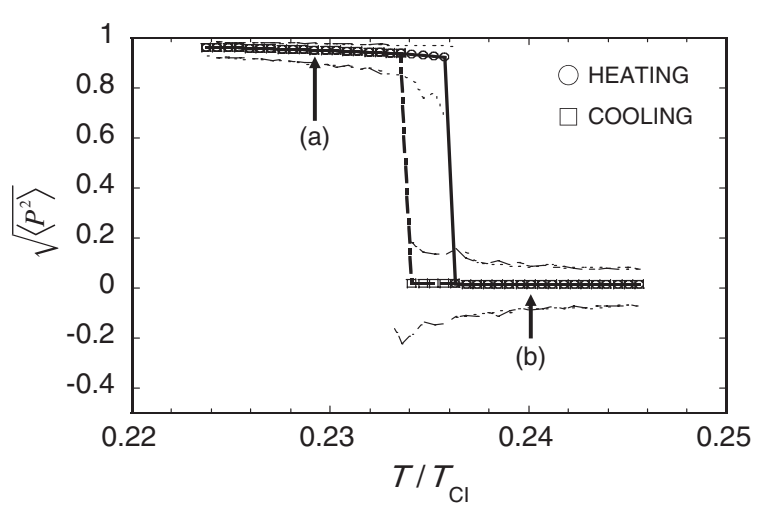

Figure 1. Temperature dependence of the spontaneous polarization for the system with $n=-1$. Open circles and squares stand for root mean squares of polarizations for heating and cooling processes, respectively. Solid and broken lines are tracing respective plots for heating and cooling processes. Light broken line for heating and chain line for cooling show the maximum and minimum envelopes of polarization fluctuation. Equilibrium snapshots at point (a) and (b) are shown by Figure 10.



Figure 2. Temperature dependence of the spontaneous polarization for the system with $n=-0.72$. The caption of Figure 1 should be referred for the meaning of marks and lines. Large fluctuation is observed around phase transition. No hysteresis appears.

$$
P=\frac{1}{N} \sum_{i=1}^{N} \sigma_{i}
$$

which varies within $-1 \leqq P \leqq 1$.

The remnant polarization of samples is usually measured as a function of temperature in experiment. In simulation, however, to trace an ensemble average of $P$ on the whole phase space is unproductive because it is zero in nature without an external bias field to positive or negative polarization. So we trace the average of squared polarization as,

$$
\begin{aligned}
\left\langle P^{2}\right\rangle & =\left\langle\left[\frac{1}{N} \sum_{i=1}^{N} \sigma_{i}\right]^{2}\right\rangle \\
& =\frac{1}{N^{2}} \sum_{i=1}^{N}\left\langle\sigma_{i}^{2}\right\rangle+\frac{2}{N^{2}} \sum_{i}^{N} \sum_{j<i}^{N}\left\langle\sigma_{i} \sigma_{j}\right\rangle
\end{aligned}
$$

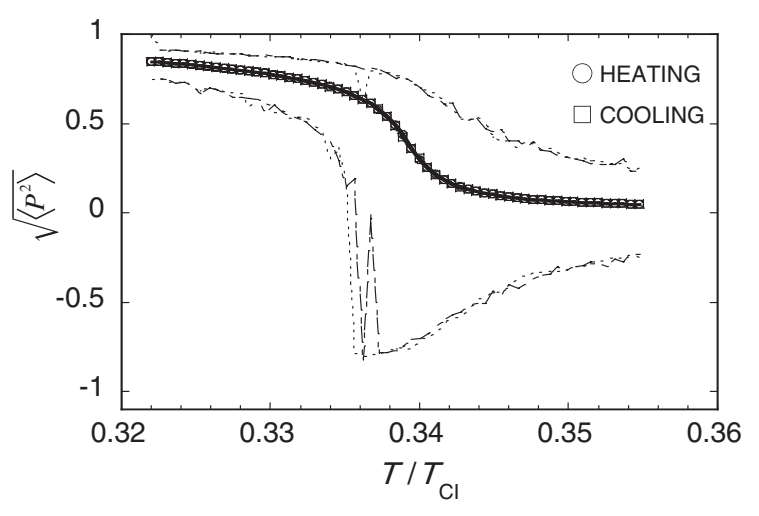

Figure 3. Temperature dependence of the spontaneous polarization for the system with $n=0$. The caption of Figure 1 should be referred for the meaning of marks and lines. Large fluctuation is observed around phase transition. No hysteresis appears.

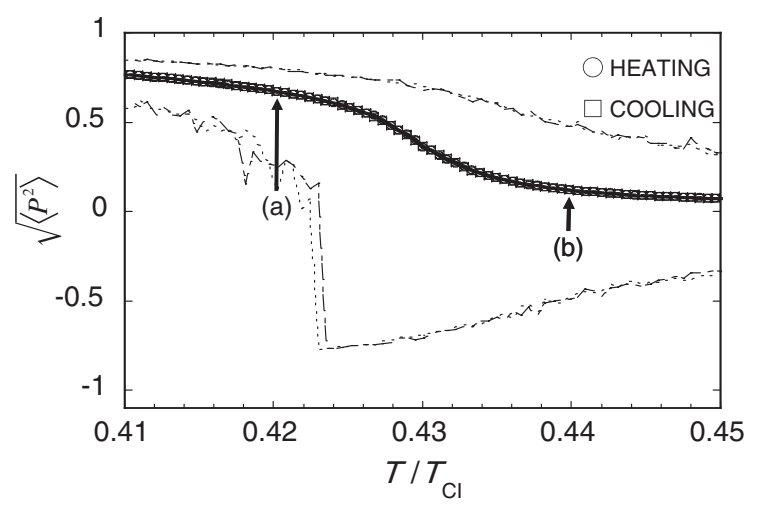

Figure 4. Temperature dependence of the spontaneous polarization for the system with $n=1$. The caption of Figure 1 should be referred for the meaning of marks and lines. Large fluctuation is observed around phase transition. No hysteresis appears. Equilibrium snapshots at point (a) and (b) are shown by Figure 11.

Here, the second term in the right hand side means correlation between $i$ and $j$ sites. When the correlation is sufficiently strong, the system becomes ferroelectric. As the correlation becomes weak, the system is transforming to paraelectric. If the second term is zero, the equation represents a random flight process and becomes

$$
\left\langle P^{2}\right\rangle \cong \frac{1}{N} \frac{2}{Z+2}
$$

Here the system is perfectly paraelectric.

Figures 1, 2, 3 and 4 illustrate temperature dependences of spontaneous polarizations for cases $n=-1$, $-0.72,0$, and 1 , respectively. The root mean squared value of the polarization and the maximum and minimum values of fluctuating polarization are traced. Temperatures on abscissa are normalized by the critical temperature $T_{\mathrm{cI}}$ of the Ising model on the two dimensional triangular lattice that was given as $k T_{\text {cl }} / V=3.64$ by Fisher. ${ }^{19}$ The Ising model corre- 


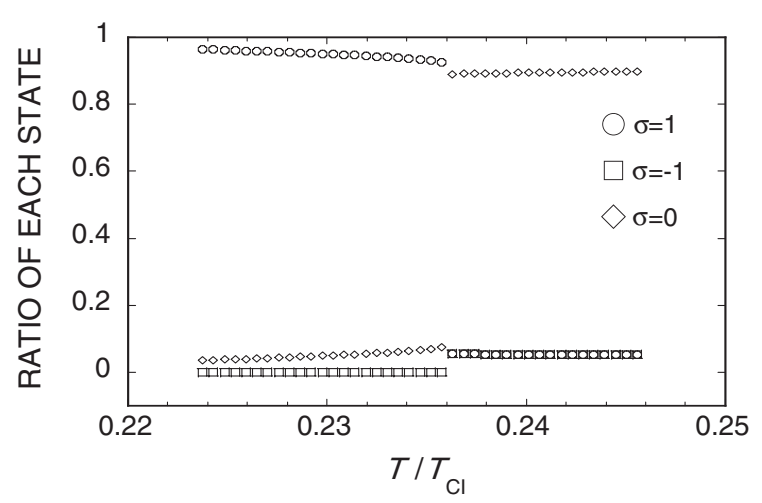

Figure 5. Temperature dependence of the population ratio of each state for the system with $n=-1$. Open circles are for $\sigma=1$, open squares for $\sigma=-1$, and open diamonds for $\sigma=0$.

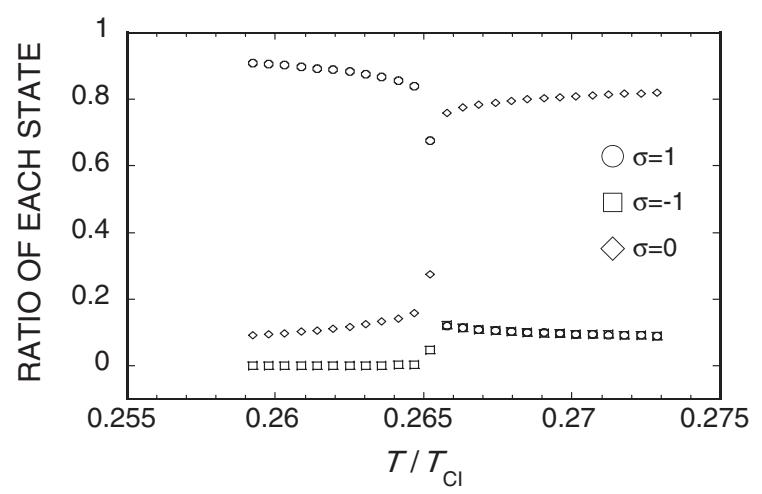

Figure 6. Temperature dependence of the population ratio of each state for the system with $n=-0.72$. The caption of Figure 5 should be referred for the meaning of marks.

sponds to the case of $n=+\infty$ in our model because the state $\sigma=0$ being needless for the Ising model can not be excited in this case as it is clear from eqs 1 and 2.

In Figure 1 the clear temperature hysteresis of polarization for $n=-1$ gives an evidence for the first order phase transition. The polarization fluctuation becomes maximum just before transitions both in the heating and the cooling process. Figure 2 shows that the temperature hysteresis of the polarization vanishes in the system of $n=-0.72$. Furthermore, the maximum and minimum envelope of the fluctuating polarization reveals that the fluctuation magnitude becomes extremely large at the transition temperature. The phase transition in this case is presumed to be in the critical region as shown by following analyses of specific heat and temperature hysteresis. Figures 3 and 4 respectively show the diffuse phase transition of the systems with $n=0$ and 1 . The temperature dependence of the polarizations is broad and temperature hysteresis is not seen in these figures. Comparison of Figure 3 with Figure 4 suggests that increase in $n$ enlarges diffuseness of the transition.

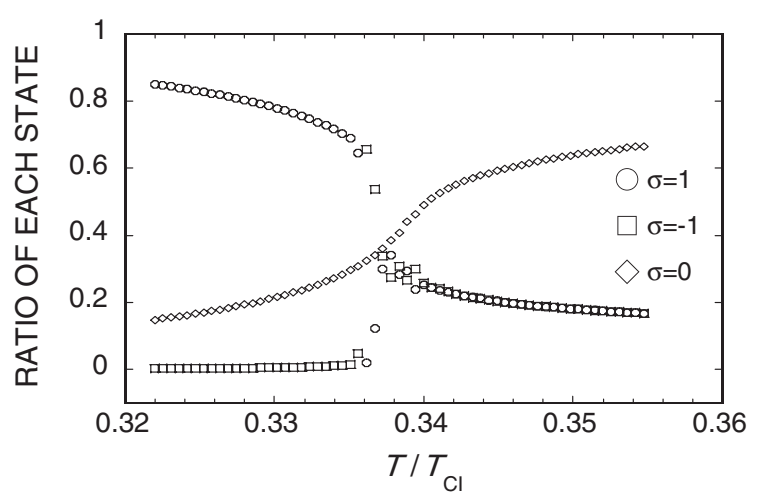

Figure 7. Temperature dependence of the population ratio of each state for the system with $n=0$. The caption of Figure 5 should be referred for the meaning of marks.

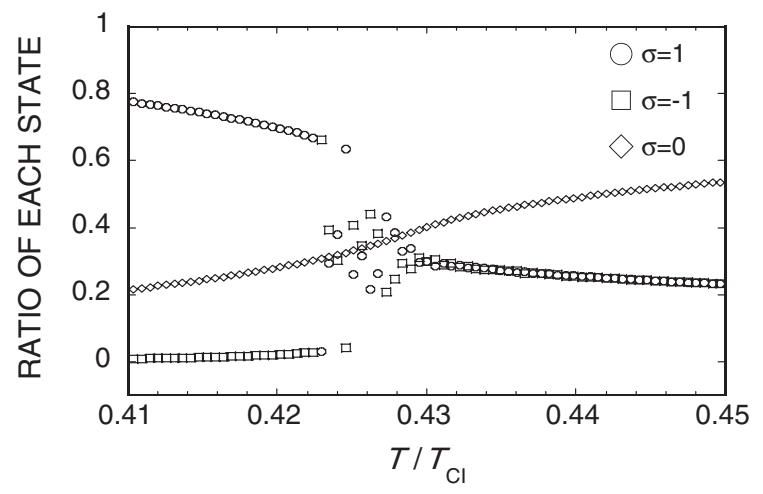

Figure 8. Temperature dependence of the population ratio of each state for the system with $n=1$. The caption of Figure 5 should be referred for the meaning of marks.

Figures 5, 6, 7 and 8 illustrate population ratios of three microscopic states of $\sigma=+1,0$ and -1 in each heating process as functions of temperature. In Figure 5 is shown the case of $n=-1$ exhibiting the first order phase transition. The ratio of each state changes drastically at the transition temperature. On the transition from ferroelectric to paraelectric, the states $\sigma=1$ and $\sigma=0$ exchange domination. Population ratios of $\sigma=1$ and $\sigma=-1$ balance in the paraelectric phase. Figure 6 illustrates the case of $n=$ -0.72 , where the transition is thought to be included in the critical region. Figures 7 and 8 illustrate that rise in the population of $\sigma=0$ with temperature becomes gentler with increase in $n$. The fact that increase in $n$ makes the transition diffuse was mentioned in an above explanation for Figures 3 and 4.

The magnitudes of fluctuating polarizations in Figures 3 and 4 and fluctuating population ratios of $\sigma=1$ and -1 in Figures 7 and 8 are broadened still far below the transition temperature. The large fluctuation comes from the situation that the whole polarization reverses in that temperature even without a bias external field. Such a polarization reversal occurs be- 
cause the system has a finite size. The reason why the reversal does not occur at lower temperatures is that the computational time is finite. Thus to trace $\langle P\rangle$ is unproductive as mentioned in the paragraph 3.1.

\section{Comparison with Experiment}

Experimental works show that when VDF mole ratio of VDF-TrFE copolymers is more than about 55\%, the phase transition thermodynamically shows the first order character and, furthermore, the intramolecular interaction for gauche conformation is negative. ${ }^{4,15,20}$ Figure 1 for $n=-1$ just corresponds to this case. When the VDF mole ratio is less than that, it shows diffuse character and the intramolecular interaction for gauche conformation is positive. Figures 3 and 4 , respectively, of $n=0$ and $n=1$ correspond to this case. Advantage or disadvantage of intramolecular interaction for gauche conformation leads the system to the first order phase transition or diffuse transition, respectively. The effect of the intramolecular interaction that suppressing conformational excitation makes the transition diffuse resemble the effect of the external magnetic field on the Ising model.

\section{Comparison with the Bethe Approximation}

In Figure 9, we compare the results provided by the

(a)

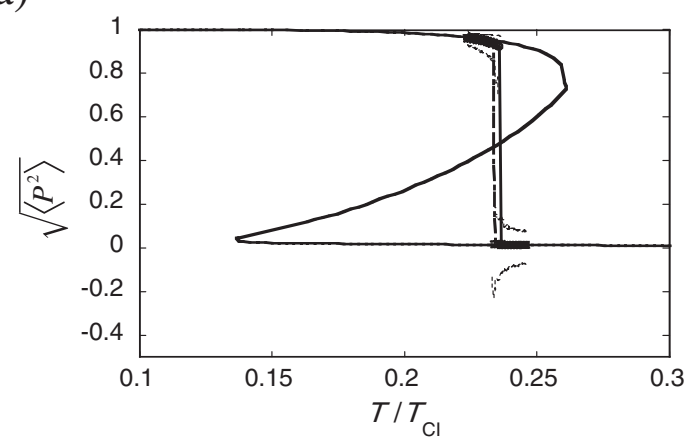

(b)

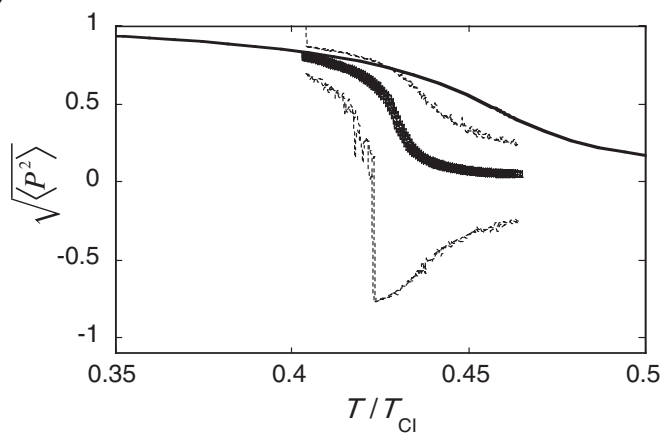

Figure 9. Temperature dependence of the spontaneous polarization in MC simulation and Bethe approximation. (a) is for $n=-1$ and (b) for $n=1$. Solid line shows result of Bethe approximation. The caption of Figure 1 should be referred for marks and broken lines. present MC simulation with the results by the Bethe approximation. ${ }^{13}$ For the comparison, we multiply the temperature in the Bethe approximation by a constant to adjust the critical temperature for $n=\infty$ to that of the Ising model.

Figures 9(a) and 9(b) indicate that the character of the phase transition expressed by the MC simulation qualitatively agrees with that by the Bethe approximation. The phase transition is of the first order for $n=-1$ in Figure 9(a), and diffuse for $n=1$ in Figure 9(b). Though the phase transition temperatures by the MC simulation and by the Bethe approximation deviate from each other, they agree on quantitative order of reversal polarization. In the graph of the Bethe approximation, the $\langle P\rangle v s$. temperature relations are plotted. This approximation is calculated by replacing a second neighbor to an average value of the system, namely, fluctuations are cut at the second neighbors. Therefore, polarization fluctuations that change the sign of $P_{\mathrm{s}}$ do not occur below the transition temperature without an external field.

\section{Equilibrium Snapshots}

The snapshots of the system for $n=-1$ are shown in Figure 10. Figure 10(a) gives a picture of the ferroelectric state at $T / T_{\mathrm{cI}}=0.229$. It illustrates a situation at (a) in Figure 1. In this snapshot, the majority is the state $\sigma=1$ and only small portion of the sites are occupied by $\sigma=0$. The state $\sigma=-1$ rarely appears. Figure 10(b) gives a picture of the paraelectric state at $T / T_{\mathrm{cI}}=0.240$ that corresponds to (b) in Figure 1. In this snapshot, the majority is $\sigma=0$. The states $\sigma=$ 1 and $\sigma=-1$ equally appear.

Figure 11 shows the snapshots of the system for $n=1$. Figure 11(a) gives a picture of the ferroelectric state at $T / T_{\mathrm{cI}}=0.420$ which corresponds to (a) in Figure 4 . This snapshot is slightly different from the ferroelectric state for $n=-1$. The state $\sigma=-1$ ap-



(a)

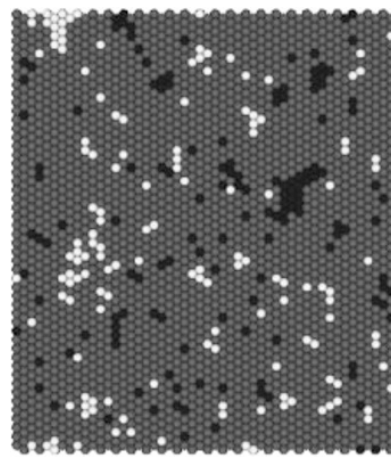

(b)
Figure 10. Snapshots of the system with $n=-1$. Lattice sites of $\sigma=1,0$ and -1 are expressed by white, gray and black circles, respectively. (a) is at $T / T_{\mathrm{cI}}=0.229$ and (b) at $T / T_{\mathrm{cI}}=0.240$. 




(a)

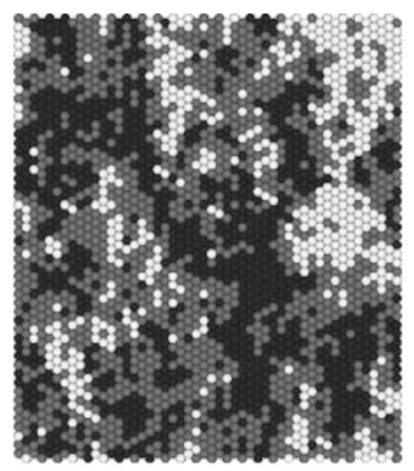

(b)
Figure 11. Snapshots of the system with $n=1$. The caption of Figure 10 should be referred for marks. (a) is at $T / T_{\mathrm{cI}}=$ 0.420 and (b) at $T / T_{\text {cI }}=0.439$.

pears for $n=1$, while it does not for $n=-1$. When $\sigma=-1$ appears, it favors to be in a cluster of $\sigma=0$ to avoid direct contact with $\sigma=1$. Figure 11(b) gives a picture of the paraelectric state at $T / T_{\mathrm{cI}}=0.439$ which corresponds to (b) in Figure 4. It indicates that the majority state $\sigma=0$ intervenes between $\sigma=1$ and $\sigma=-1$ and plays a role of weakening interaction between them.

\section{Specific Heat and Critical Character}

We have suggested the existence of the second order phase transition between the first order phase transition and the diffuse transition. It is next to impossible in a real finite system to verify the critical second order phase transition by observing critical divergence of the second derivative of free energy. However, we find the critical character of the system with a value of $n$ by means of calculating the specific heat of the system. ${ }^{21}$ The specific heat is estimated as follows.

$$
C v=\frac{1}{N k T^{2}}\left(\left\langle H^{2}\right\rangle-\langle H\rangle^{2}\right)
$$

Calculated specific heats are illustrated in Figure 12. A high and sharp peak is seen between peaks with the temperature hysteresis and low peaks accompanied by diffuseness. Figure 13 shows the temperature hysteresis estimated from the difference between peak temperatures in the heating and the cooling process and peak heights of the specific heats as functions of the interaction ratio. At the same value of $n$ that is approximatly -0.74 , the temperature hysteresis vanishes and the highest peak of the specific heat is observed. This point is thought to specify the critical point where the second order phase transition occurs.

Ising model derives the second order phase transition. If the ferroelectric polymer system took only planar zigzag conformation, the model abstracting an essence of the phenomenon would be the Ising model. However, the paraelectric phase of the polymer is re-

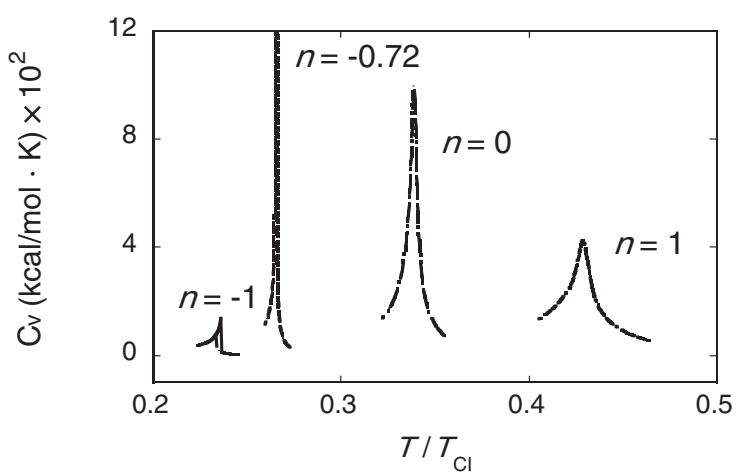

Figure 12. Temperature dependence of the specific heat of the system with interactions specified in the figure. Only the specific heat with $n=-1$ has temperature hysteresis. In Figures 12 and $13, V$ is taken as 1 .



Figure 13. Interaction ratio dependence of the height of the specific heat at the phase transition temperatures $\left(T_{\mathrm{c}}\right)$ and the temperature hysteresis $\left(1-T_{\mathrm{c}}(\right.$ Cooling $) / T_{\mathrm{c}}($ Heating $\left.)\right)$.

alized by distinction of spontaneous polarization due to including random helical conformations in addition to cancellation of dipole moment due to antiparallel alignment. Then we would make the three states model. We can find a series of phase transitions from the first order to diffuse through the second order phase transition.

\section{Phase Transformation}

In order to follow the detail of the phase transformation, we calculate the probability density of the system as a function of polarization $\Psi(P)$. It is normalized as

$$
\int_{-1}^{1} \psi(P) \mathrm{d} P=1
$$

Figure 14 shows the temperature dependence of $\Psi(P)$ during heating for $n=-1$ and $n=1$ obtained by integrating $5 \times 10^{6}$ MCS's not less than $5 \times 10^{6}$ MCS at each temperature. When the temperature is far from the phase transition, both distributions are localized around a single peak near $P=1$. In the process of $n=-1$ giving the first order phase transition in 
(a)

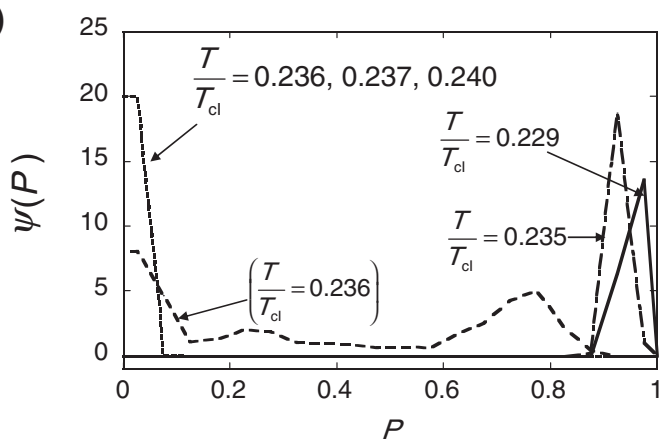

(b)

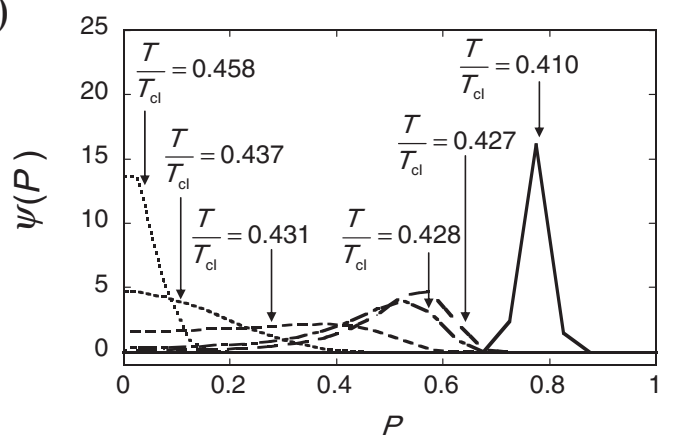

Figure 14. The probability distribution of spontaneous polarization depending on temperature. (a) is for $n=-1$ and (b) for $n=1$. Temperatures of distributions are denoted in the figure. The only distribution designated with $\left(T / T_{\mathrm{cI}}=0.236\right)$ was obtained by an average of $10^{4}$ MCS's from $2.33 \times 10^{6}$ to $2.34 \times$ $10^{6}$ MCS. The others are averages of $10^{6}$ MCS's in the latter half at each temperature.

Figure 14(a), the single peak localized near $P=1$ shifts to $P=0$ with narrow temperature change. The transformation is attained in an instant. The only distribution designated with $\left(T / T_{\mathrm{cI}}=0.236\right)$ in the figure was obtained by an average of $10^{4}$ MCS's from $2.33 \times 10^{6}$ to $2.34 \times 10^{6}$ MCS. Double peaks are just observed near $P=0$ and 1 . The distribution does not approach $P=0$ gradually, but the peak at $P=0$ rises simultaneously with the decrease in the peak height near $P=1$. The average of $5 \times 10^{6}$ MCS's in the latter half is the same with that at $T / T_{\mathrm{cI}}=0.237$. On the other hand, in Figure 14(b) giving the diffuse transition, $\Psi(P)$ has a widely distributed shape in the transition region. When temperature increases from ferroelectric range to paraelectric, the peaks broaden continuously getting together to $P=0$ above the transition temperature. From these distinguishable characters of the phase transition behavior we presume that the first order phase transition of this system has a type of nucleation and growth and the diffuse transition occurs through spinodal decomposition. ${ }^{22}$ These mechanisms of phase transformation, however, must be confirmed by simulation tracing temporal change of the system. We leave the detailed study as a future work.

\section{CONCLUSIONS}

The ferreoelectric phase transition of the simple three-states model accompanied by conformational transformation of molecular chains was simulated by the MC method. It was confirmed that the phase transition of the model changes the thermodynamical character from the first order phase transition to the diffuse transition with an increase in the intramolecular interaction. The specific heat was derived from the energy fluctuation of the system. The peak of the specific heat observed at the transition temperature becomes the maximum in the system where the temperature hysteresis of the phase transition vanishes. It suggests the existence of the second order critical transition between the first order transition and the diffuse transition. Furthermore, the transformation mechanism of the phase transition was proposed. The system exhibiting the first order phase transition transforms the phase due to nucleation and growth and that doing the diffuse transition transforms the phase due to spinodal decomposition.

Acknowledgment. This work was supported by Grants-in-Aid from the Ministry of Education, Culture, Sports, Science and Technology of Japan, No. 18068003 (Priority Area "Soft Matter Physics") and No. 17310058.

\section{REFERENCES}

1. R. C. G. Naber, C. Tanase, P. W. M. Blom, G. H. Gelinck, A. W. Marsman, F. J. Touwslager, S. Setayesh, and D. M. de Leeuw, Nature Mater., 4, 243 (2005).

2. K. N. N. Unni, R. de Bettignies, S. Dabos-Seignon, and J.-M. Nunzi, Appl. Phys. Lett., 85, 1823 (2004).

3. T. Nakajima, R. Abe, Y. Takahashi, and T. Furukawa, Jpn. J. Appl. Phys., Part 2, 44, L1385 (2005).

4. T. Furukawa, Phase Transitions, 18, 143 (1989).

5. S. Ikeda, H. Kominami, K. Koyama, and Y. Wada, J. Appl. Phys., 62, 3339 (1987).

6. Y. Takahashi, H. Kodama, M. Nakamura, T. Furukawa, and M. Date, Polym. J., 31, 263 (1999).

7. H. Kodama, Y. Takahashi, and T. Furukawa, Jpn. J. Appl. Phys., Part 1, 38, 3589 (1999).

8. A. Odajima, Ferroelectrics, 57, 159 (1984).

9. R. Zhang and P. L. Taylor, J. Appl. Phys., 73, 1395 (1993).

10. Y. Abe, K. Tashiro, and M. Kobayashi, Comput. Theor. Polym. Sci., 10, 323 (2000).

11. Y. Abe and K. Tashiro, Polymer, 42, 3409, 9671 (2001).

12. Y. Abe and K. Tashiro, J. Polym. Sci., Part B: Polym. Phys., 39, 689 (2001).

13. S. Ikeda and H. Suda, Phys. Rev. E: Stat., Nonlinear, Soft. Matter Phys., 56, 3231 (1997).

14. S. Ikeda and T. Yuki, Polymer, 43, 4783 (2002).

15. K. Tashiro, K. Takano, M. Kobayashi, Y. Chatani, and H. 


\section{FUKUZAWA et al.}

Tadokoro, Ferroelectrics, 57, 297 (1984).

16. N. Metropolis, A. W. Rosenbluth, M. N. Rosenbluth, A. H. Teller, and E. Teller, J. Chem. Phys., 21, 1087 (1953).

17. D. P. Landau, Phys. Rev. B: Condens. Matter Mater. Phys., 13, 2997 (1976).

18. M. E. J. Newman and G. T. Barkema, in "Monte Carlo Methods in Statistical Physics" Clarendon Press, Oxford,
1999, p 45.

19. M. E. Fisher, Rep. Prog. Phys., 30, 615 (1967).

20. K. Koga and H. Ohigashi, J. Appl. Phys., 59, 2142 (1986).

21. D. Fukuzawa, A. Nishioka, T. Koda, and S. Ikeda, Ferroelectrics, 338, 153 (2006).

22. R. Kurita and H. Tanaka, Science, 306, 845 (2004). 